\title{
Towards the Development of Orally Available Peptide Therapeutics
}

\author{
Xu-Dong Kong ${ }^{\mathrm{a}}$ and Christian Heinis ${ }^{* b}$
}

\begin{abstract}
Peptides have a number of attractive properties that make them an interesting modality for drug development, including their ability to bind challenging targets, their high target specificity, and their non-toxic metabolic products. However, a major limitation of peptides as drugs is their typically poor oral availability, hindering their convenient and flexible application as pills. Of the more than 60 approved peptide drugs, the large majority is not orally applicable. The oral delivery of peptides is hampered by their metabolic instability and/or limited intestinal uptake. In this article, we review the barriers peptides need to overcome after their oral administration to reach disease targets, we highlight two recent successes of pharma companies in developing orally applicable peptide drugs, and we discuss efforts of our laboratory towards the generation of bioavailable cyclic peptides.
\end{abstract}

Keywords: Combinatorial library · Cyclic peptide · Drug development · Macrocycle $\cdot$ Phage display

\section{Attractiveness of Peptides as Therapeutics}

Peptides combine a number of qualities that make them an attractive modality for drug development. They can bind to proteins with high affinity and selectivity, they can engage with 'challenging' targets such as proteins having flat and featureless surfaces, they are suited to block protein-protein interactions, and their metabolic products typically have no or low toxicity (often amino acids). A further strength of peptide drugs is their accessibility to chemical synthesis, which gives many options in the development and optimization of drug leads and can facilitate their production. Finally, peptides are amenable to biological display methods such as phage or mRNA display that allow for the screening of billions of random peptide sequences and an efficient development of bioactive peptides from scratch. Over 60 peptides are clinically approved and are used for the treatment of infectious diseases, cancer, cardiovascular diseases and other maladies. ${ }^{[1,2]}$ Many peptide drugs have properties and therapeutic activities that cannot be easily achieved by other molecule formats.

Unarguably the largest weakness of peptide therapeutics is their typically low oral availability. Only a few of the approved peptides can be applied by the oral route, such exceptions being the antidiuretic desmopressin, the immunosuppressant cyclosporine, and the diabetes drug semaglutide. ${ }^{[3,4]}$ The bioavailability of the three drugs differs strongly, ranging from around $30 \%$ for cyclosporine to around $0.1 \%$ for desmopressin, the low value of the latter drug being acceptable due to its high potency. Most other peptide drugs require parenteral administration, which can be a deterrent for medication adherence due to aversion to injections, discomfort at the injection site, and sometimes need for help from health professionals.

\section{Barriers for Orally Applied Peptides}

The limited oral availability of peptides is mainly due to three hurdles that are difficult to overcome, the first one being proteases in the gastrointestinal (GI) tract that degrade peptides, the second one being poor crossing of the epithelial layer due to the large size and polar surface, and the third one being first-pass metabolism in the liver (Fig. 1). ${ }^{[3]}$ The proteolytic degradation starts in the stomach where pepsin degrades peptides at low $\mathrm{pH}$, and continues in the small intestine where mainly trypsin and chymotrypsin degrade peptides at neutral $\mathrm{pH}$. Simply said, our bodies recognize peptides as food when ingested. A study assessing the stability of peptides in the GI tract showed that for most peptides, the proteolytic pressure is highest in the small intestine. [5] The second hurdle for orally applied peptides is the crossing of the epithelial cell layer to enter blood vessels. The correlation between oral uptake and the peptides' physicochemical properties was studied in detail and valuable rules and guidelines are now available. ${ }^{[4,6]}$ Most peptides showing a reasonably high bioavailability have a molecular weight well below one kilodalton, fewer than five hydrogen bond donor groups, and a polar surface area smaller than $250 \AA^{2}$. The third hurdle that orally applied peptides need to overcome is the first pass metabolism. Once a peptide is absorbed in the digestive tract, it flows through arterioles into the liver where it may be metabolized by hepatic enzymes. To develop orally available drugs, all the three barriers described need to be passed.

\section{Recent Successes in the Development of Oral Peptide Drugs}

Extensive efforts have been made to convert therapeutically active peptides into orally available drugs. The peptides are typically engineered in multiple cycles of structure modification and characterization to fulfill the above-described requirements such as high proteolytic stability and small size/polar surface for gut absorption. Peptides are engineered for stability by altering the amino acid sequence eliminating vulnerable sites, by cyclizing or stapling to impose conformational constrains onto the backbone and hindering access of proteases, or by overall reducing the peptidic nature by replacing amino acids and peptide bonds by other chemical groups and linkages. In addition to these molecule engineering and classical medicinal chemistry approaches, formulation strategies are applied to facilitate protease resistance 


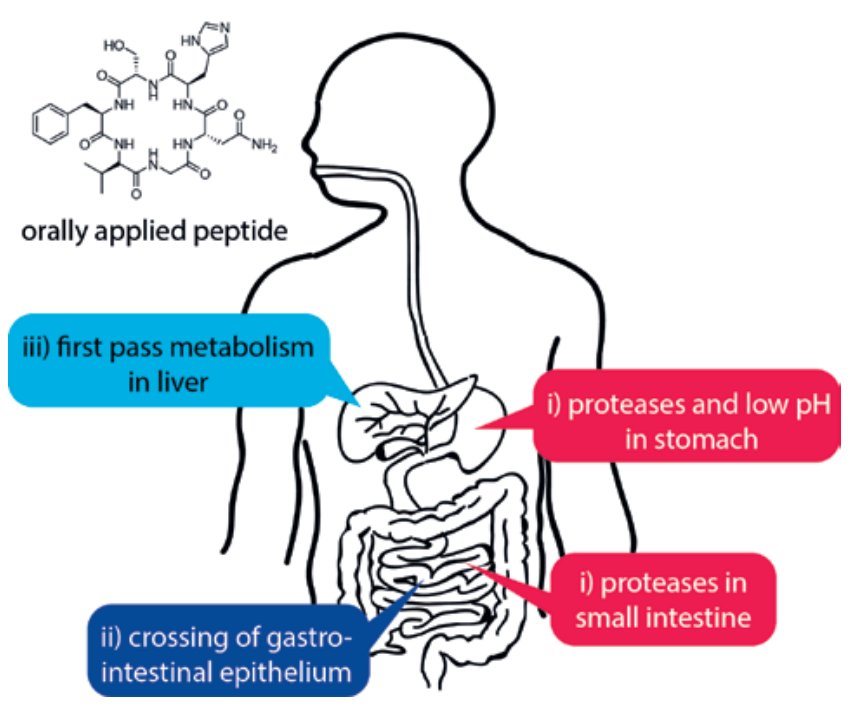

Fig. 1. Barriers for oral peptide drugs: i) degradation by proteases in the stomach or intestine, ii) crossing of the epithelial layer, and iii) first-pass metabolism in the liver. For drugs that act locally in the first layers of the gastrointestinal tract (e.g. bacterial infections, inflammation of bowel), only the first barrier, the proteolytic degradation, needs to be overcome. For drugs that need to be delivered systemically, all three barriers need to be passed.

or epithelia absorption, including co-administration with inhibitors of gut enzymes and application of excipients for enhancing mucus penetration or intestinal absorption.

Two recent successes in developing orally applicable peptide drugs are highlighted in the following, the first one being the development of the anti-constipation drug Linaclotide that was approved in 2012 and has reached net sales of over one billion US\$ in 2020, and the second one being the oral formulation of the glucagon-like peptide 1 (GLP-1) receptor agonist semaglutide approved in 2019. Linaclotide is a 14-amino acid peptide that contains six cysteines which are connected by three disulfide bridges to constrain the peptide's skeleton, hindering its degradation by proteases in the gastrointestinal tract. ${ }^{[7]}$ The peptide activates the cell surface receptor of guanylate cyclase $2 \mathrm{C}$ leading to an increase in intestinal fluid that is beneficial in the treatment of irritable bowel syndrome with constipation and chronic constipation. After oral application, Linaclotide acts in the lumen of the GI tract and thus needs to resist proteolytic degradation after oral application, but does not need to be absorbed. Semaglutide is a GLP-1 receptor agonist that potentiates meal-stimulated insulin release. ${ }^{[8]}$ It is chemically similar to GLP-1 but has modifications that prevent breakdown by dipeptidyl peptidase- 4 and prolong the half-life through albumin binding. It was initially developed and approved as a once-weekly injectable drug for type 2 diabetes, and was recently developed further for oral delivery through formulation in a tablet containing the permeation enhancer salcaprozate sodium (SNAC). In 2019, the oral semaglutide was approved for controlling blood sugar in adult patients with type 2 diabetes, and represents a landmark in the development of oral peptide therapeutics.

\section{In vitro Evolution of Peptides that Resist Gastrointestinal Proteolysis}

As described above, the first barrier for orally applied peptides is the high proteolytic pressure in the gastrointestinal tract. For many bioactive peptides or approved peptide drugs, it has been difficult to impossible to generate variants that are sufficiently stable to resist GI proteases while retaining their biological activity. Given these challenges, our laboratory has asked the question if it was possible to use phage display to isolate cyclic peptides that are both target-specific and protease-stable from random libraries. We proposed the approach shown in Fig. 2a that is adapted from previously developed methods to evolve proteolytically stable proteins by phage display. ${ }^{[9,10]}$ In this scheme, billions of random, linear peptides are genetically encoded by phage display, then cyclized by bridging pairs of cysteines by chemical linkers, and finally subjected to two sequential selection events, the first one for enriching protease-stable peptides (i.e. by eliminating the labile ones) and the second one for capturing those that bind with high affinity to a protein target of interest.[11,12] We used peptides containing four cysteines that were cyclized by two chemical bridges (Fig. 2a, left graphic), expecting that the conformational constrains would hinder binding to the proteases' active sites for some of the peptide formats.[13]

The in vitro evolution of proteolytically stable peptides required phage particles that resist GI-relevant protease concentrations. Our technical problem was that the disulfide-free phages, which we had previously applied for the selection of bicyclic peptides ${ }^{[14]}$ and double bridged peptides, ${ }^{[13]}$ survive only if the protease concentration of porcine intestinal fluid is 500-fold diluted. ${ }^{[11]}$ Wild-type fd phage resists undiluted intestinal fluid for at least 30 mins at $37^{\circ} \mathrm{C}$ but contains six cysteines in the p3 phage coat protein (three disulfide bridges) that can interfere with the chemical cyclization reaction applied to displayed peptides. Toward the use of wild-type phage, we searched and found thiol-reduction conditions to reduce disulfide-bridged cysteines in the displayed peptides before reacting them with cyclization reagents, while leaving the six disulfide-forming cysteines in the wild-type p3 coat protein mostly untouched.[12]

We have applied the approach to the blood coagulation factor FXIa as a first model target. From a library of $>10$ billion different peptides, we were able to isolate a cyclic peptide that bound with nanomolar affinity to the active site of FXIa $\left(K_{\mathrm{i}}=19 \pm 2 \mathrm{nM}\right)$ and that was highly stable in porcine gastric and intestinal fluids for extended time periods (half-life of $>2 \mathrm{~h}$ ). We studied the reasons for the high stability by solving the X-ray structure (Fig. 2b) and testing the stability of peptide variants with only one or no chemical bridges, and found that both the conformational constrains imposed by chemical bridges and the specific amino acid sequence were essential. Application of a highly stable identified peptide to mice via the oral route and analysis of the residual peptide after different time points showed that it remained largely intact in all segments of the gastrointestinal tract, including the highly acidic stomach and the small intestine, which exert enormous proteolytic pressure. ${ }^{[12]}$ Unfortunately, only a very small fraction of the peptide, $0.26 \%$, reached the blood stream, most likely because the 9-amino acid peptide was too large and polar (one positive charge) to cross efficiently the epithelium.

While the peptides isolated with the phage display approach did not reach the blood stream efficiently, the approach can readily be used to generate orally applicable peptides that act locally on targets in the lumen of the GI tract, as for example the above described nature-derived peptide drug linaclotide. With the goal of developing peptides that can be taken as a pill and act locally in the GI tract, we have applied the phage display approach to generate proteaseresistant cyclic peptide antagonists of interleukin-23 (IL-23) receptor, a target implicated in Crohn's disease and ulcerative colitis, two inflammatory diseases of the digestive tract. Phage selections yielded peptide ligands with high proteolytic stability for this target too. ${ }^{[12]}$ Additional diseases and targets that may be addressed with orally applied protease-stable peptides could be tumors in organs of the GI tract or bacterial infections of the gut.

\section{High-throughput Screening of Sub-kilodalton Cyclic Peptides}

To treat diseases systemically, the orally applied peptide drugs need to be absorbed in the GI tract. As described above, the com- 
a

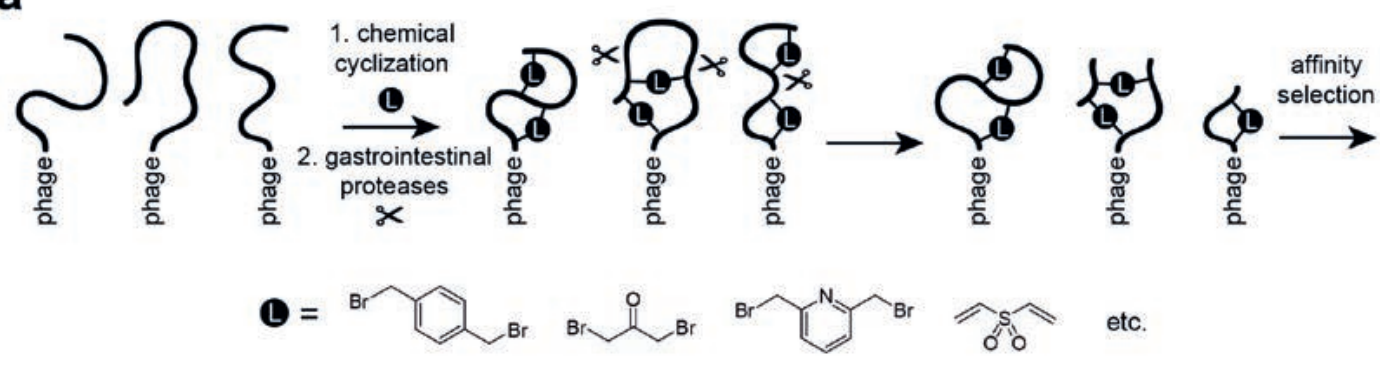

b

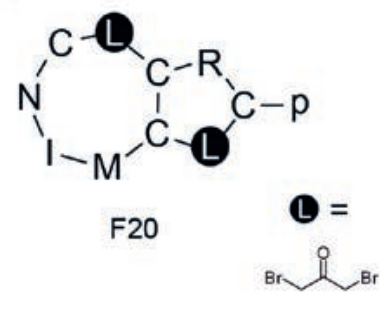

Fig. 2. Phage display selection of protease-resistant, target-specific cyclic peptides. (a) Phage display selection procedure. Phageencoded linear peptides are first converted into 'double-bridged peptides' in chemical reactions using bis-electrophile cyclization reagents $(L)$ that cross-link pairs of cysteines. The resulting library is exposed to pancreatic proteases to eliminate unstable peptides and then subjected to affinity selections using immobilized target protein. (b) Structure of the coagulation factor FXI inhibitor F20 that was developed with the approach in (a) and that survived oral application in mice. The peptide sequence and cysteine connectivity are shown on the left and the X-ray structure of a parental peptide of F20 in the middle and on the right.

Fig. 3. Synthesis and screening approach for the development of sub-kilodalton cyclic peptides. (a) Schematic depiction of the combinatorial approach. A panel of $n$ short peptides (gray) are combinatorially cyclized with $\mathrm{m}$ cyclization linkers (red) to yield $\mathrm{m} \times \mathrm{n}$ cyclic peptides. The cyclization linkers are bis-electrophile reagents that connect a thiol and an amino group present in each peptide. Due to the high-yielding reactions, the products are screened for target engagement
C<smiles>N=C(N)NCCCC(NC(=O)CC1CCCN1C(=O)CN(Cc1ccccc1CO)Cc1ccccc1CSCC(NC(=O)O)C(N)=O)C(=O)O</smiles>
$42 \pm 5 \mathrm{nM}$ without purification, allowing a large throughput. (b) Result of a screen to identify cyclic peptide inhibitors of thrombin. The darker the color, the better was thrombin inhibited by a cyclic peptide. (c) Identified thrombin inhibitor P2. The cyclization linker is shown in red. (d) X-ray structure of P2 bound to thrombin. pounds must fulfil several physicochemical properties in order to be absorbed into the blood stream, and the key criteria are size and polarity. Overall, cyclic peptides having up to around six amino acids and a limited polar surface have a reasonably good chance to be absorbed. The development of such short cyclic peptides by phage display is difficult because the library size would be limited to less than a million cyclic peptides, as only the 20 natural amino acids can be used as building blocks, and two of the amino 
acid positions are required for cyclization. Innovative strategies based on mRNA ${ }^{[15]}$ or phage display ${ }^{[16,17]}$ allow the incorporation of unnatural amino acids, but the number of different amino acids that can simultaneously be incorporated is limited, restricting the number of potential sequences in the case of short peptides.

Given the limitations of biological display techniques for the generation of sub-kilodalton cyclic peptides, our laboratory has moved to the development of cyclic peptide synthesis strategies based on synthetic building blocks such as Fmoc amino acids. The commercial availability of hundreds of diverse unnatural amino acid building blocks allows, in principle, the synthesis of large combinatorial libraries of small cyclic peptides having an enormous chemical and structural diversity. To generate such libraries, we proposed to combinatorially cyclize in individual wells of microwell plates $m$ random peptides with $n$ chemical linkers to obtain $m \times n$ macrocyclic compounds and to screen them without purification (Fig. 3a). The omission of a purification step promised access to large libraries of ten-thousands of macrocyclic compounds with a moderate effort, but it required efficient macrocyclization reactions so that the main product in each well was the desired macrocyclic compound.

We found that many of the bis-electrophile reagents that we had used for the generation of double-bridged peptides by phage display (shown in Fig. 2a) were suited for cyclizing peptides via a thiol group and an amino group (Fig. 3a). ${ }^{[18]}$ The yields were high for a wide range of different peptide sequences and bis-electrophile linkers. We thus applied such reactions to build a macrocycle library of 8988 compounds and screened it against two targets, tissue kallikrein 5 and thrombin, the result of the latter screen being shown in Fig. 3b. ${ }^{[18]} \mathrm{X}$-ray structure analysis of the best thrombin inhibitor, $\mathbf{P 2}\left(K_{\mathrm{i}}=42 \mathrm{nM}\right.$; Fig. 3c) revealed that the macrocycle perfectly fits to the active site of the protease (Fig. $3 \mathrm{~d}$ ). This proof-of-principle work showed that large libraries of sub-kilodalton macrocyclic compounds can be synthesized and screened with this new approach. While the thrombin inhibitor P2 is positively charged and thus not orally applicable, the approach can be applied for generating and screening ten- to hundred-thousands of small cyclic peptides having a small polar surface and high chances of being bioavailable.

\section{Acknowledgements}

We thank the Swiss National Science Foundation for supporting the research described in the last two chapters (project grant 192368 and NCCR Chemical Biology).
[1] J. L. Lau, M. K. Dunn, Bioorg. Med. Chem. 2018, 26, 2700, https://doi.org/10.1016/j.bmc.2017.06.052.

[2] A. Zorzi, K. Deyle, C. Heinis, Curr. Opin. Chem. Biol. 2017, 38, 24, https://doi.org/10.1016/j.cbpa.2017.02.006.

[3] D. J. Drucker, Nat. Rev. Drug Discov. 2020, 19, 277, https://doi.org/10.1038/s41573-019-0053-0.

[4] D. S. Nielsen, N. E. Shepherd, W. Xu, A. J. Lucke, M J. Stoermer, D. P. Fairlie, Chem. Rev. 2017, 117, 8094, https://doi.org/10.1021/acs.chemrev.6b00838.

[5] J. Wang, V. Yadav, A. L. Smart, S. Tajiri, A. W. Basit, Mol. Pharm. 2015, 12, 966, https://doi.org/10.1021/mp500809f.

[6] F. Giordanetto, J. Kihlberg, J. Med. Chem. 2014, 57, 278, https://doi.org/10.1021/jm400887j.

[7] P. L. McCormack, Drugs 2014, 74, 53, https://doi.org/10.1007/s40265-013-0157-5.

[8] L. B. Knudsen, J. Lau, Front. Endocrinol. (Lausanne) 2019, 10, https://doi.org/10.3389/fendo.2019.00155.

[9] P. Kristensen, G. Winter, Fold. Des. 1998, 3, 321, https://doi.org/10.1016/S1359-0278(98)00044-3.

[10] V. Sieber, A. Plückthun, F. X. Schmid, Nat. Biotechnol. 1998, 16, 955 , https://doi.org/10.1038/nbt1098-955.

[11] V. Baeriswyl, C. Heinis, Protein Eng. Des. Sel. 2013, 26, 81, https://doi.org/10.1093/protein/gzs085.

[12] X.-D. Kong, J. Moriya, V. Carle, F. Pojer, L. A. Abriata, K. Deyle, C. Heinis, Nat. Biomed. Eng. 2020, 4, 560, https://doi.org/10.1038/s41551-020-0556-3.

[13] S. S. Kale, C. Villequey, X.-D. Kong, A. Zorzi, K. Deyle, C. Heinis, Nat. Chem. 2018, 10, 715, https://doi.org/10.1038/s41557-018-0042-7.

[14] C. Heinis, T. Rutherford, S. Freund, G. Winter, Nat. Chem. Biol. 2009, 5 , 502, https://doi.org/10.1038/nchembio.184.

[15] T. Passioura, W. Liu, D. Dunkelmann, T. Higuchi, H. Suga, J. Am. Chem Soc. 2018, 140, 11551, https://doi.org/10.1021/jacs.8b03367.

[16] A. E. Owens, J. A. Iannuzzelli, Y. Gu, R. Fasan, ACS Cent. Sci. 2020, 6, 368, https://doi.org/10.1021/acscentsci.9b00927.

[17] B. Oller-Salvia, J. W. Chin, Angew. Chemie Int. Ed. 2019, 58, 10844 https://doi.org/10.1002/anie.201902658.

[18] S. S. Kale, M. Bergeron-Brlek, Y. Wu, M. G. Kumar, M. V. Pham, J. Bortoli, J. Vesin, X.-D. Kong, J. F. Machado, K. Deyle, P. Gonschorek, G. Turcatti, L. Cendron, A. Angelini, C. Heinis, Sci. Adv. 2019, 5, eaaw2851, https://doi.org/10.1126/sciadv.aaw2851.

\section{License and Terms}

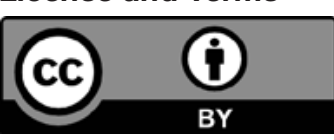

This is an Open Access article under the terms of the Creative Commons Attribution License CC BY 4.0. The material may not be used for commercial purposes.

The license is subject to the CHIMIA terms and conditions: (http:// chimia.ch/component/sppagebuilder/?view=page \&id=12).

The definitive version of this article is the electronic one that can be found at https://doi.org/10.2533/chimia.2021.514 